\title{
AN INDUSTRIAL INSPECTION APPROACH FOR WELD DEFECTS USING MACHINE LEARNING ALGORITHM
}

\author{
Kalai Selvi V, \\ Department of Computer Science and Engineering, \\ Hindustan University, \\ Kelambakkam, Chennai, India \\ pvkselvi@yahoo.com \\ Dr. John Aravindar D, \\ Department of Computer Science and Engineering, \\ Hindustan University, \\ Kelambakkam, Chennai, India \\ jaravindhar@hindustanuniv.ac.in
}

\begin{abstract}
The weld defects are formed due to the incorrect welding patterns or wrong welding process. The defects in the weld may vary from size, shape and their projected quality. The most common weld defects occur during welding process is slag inclusions, porosity, lack of fusion and incomplete penetration. In this study, an effective method for weld defect classification using machine learning algorithm is presented. The system uses Speeded-up Robust Features (SURF) for feature extraction and one of the machine learning algorithms called Auto-Encoder Classifier (AEC) for classification. Initially, the features that distinguish weld defects and no defects in the weld image are extracted by SURF. Then, AEC is analyzed for weld image classification using different number of neurons in different hidden layers (2 and 3 hidden layers). The performance of the system is evaluated by GD X-ray weld image database. The results show that the weld images are correctly classified with $98 \%$ accuracy using SURF and AEC.
\end{abstract}

Keywords: X-ray weld images, weld image classification, speeded-up robust features, machine learning, auto-encoder classifier.

\section{INTRODUCTION}

Welding image classification in low rank representation using spatial pyramid matching is discussed in [1]. The input weld image features are extracted by Scale Invariant Feature Transform (SIFT). The encoding is made by vector quantization, sparse representation and low rank representation. Then the classification is made by linear and non-linear Support Vector Machine (SVM). Gaussian kernel and Convolutional Neural Network (CNN) based welding defect classification is discussed in [2]. Gaussian kernel is used to blur the image. Features are extracted and classified by CNN in two stages.

A classification method for arc weld image based on invariant moments is presented in [3]. Features are extracted by using seven Hu moment invariants and then similarity parameter is constructed. Finally, the classification is made by similarity parameter. The classification of linear welding defects using image processing techniques is discussed in [4]. At first, filtering and segmentation are employed to extract the defect regions. Then, the weld defect images are classified into incomplete fusion, lack of penetration and external undercut with SVM and k-nearest neighbour classifier.

Weld defect detection using c-means segmentation method is described in [5]. Initially, wiener filter is used in the preprocessing stage to remove noise. Then, 
c-means technique is used for segmentation. The connected component labeling technique is used to detect the contour region. Weld defect classification using radiographic images is discussed in [6] based on SVM classifier. In the preprocessing stage, region of interest is extracted at first. Then, segmentation is made by thresholding, clustering techniques and background subtraction method. Features like elongation, eccentricity, compactness, symmetry and rectangularity features and invariant Hu moments are also extracted. Finally, the classification is made by SVM classifier.

Weld defect classification using gentle Adaboost algorithm is discussed in [7]. From the weld images, features are extracted by moment-based features. The defect classes like porosity, lack of penetration, crack and solid inclusion are classified using gentle Adaboost classifier. Weld defect classification and detection using geometric features is discussed in [8]. In the pre-processing stage, median and non-linear filters are used for noise reduction. The edges are detected by canny edge detector. The sauvola thresholding and morphological operations are used for segmentation. The geometric features like major axis, minor axis, area, perimeter, solidity, orientation, convex area and eccentricity are extracted. At last, the classification is made by Artificial Neural Network (ANN).

The classification of flaws in radiographic weld images using ANN is discussed in [9]. Initially, wiener filter is used in the preprocessing stage to remove noises. The region, edge and watershed segmentation techniques are used for segmentation. Then, the features are extracted by texture and geometric features. The classification of flaws is made by using ANN. Deep learning and fuzzy classification based welding monitoring system is discussed in [10]. The deep learning method is used for feature extraction. Finally, fuzzy rule based classification algorithm is used for the classification.

Welding defects classification based on binary tree multi-classifier is discussed in [11]. Six parameters are extracted from the characteristics of weld images as features. Then, binary tree based multiclass SVM is used for classification. SVM based classification of welding defects is described in [12] using radiographic images. The five features like edge smoothness, aspect ratio, ends morphological change, coordinate of barycenter to weld center and regional intensity are extracted. The classification is made by SVM based binary decision tree.

In this study, an industrial inspection approach for weld defects using machine learning algorithm is presented. The organization of this paper is as follows: Materials and methods used for weld image classification system are described in section 2. Experimental results and discussion of weld image classification system is explained in section 3 . Section 4 concludes the weld image classification system using SURF and AEC.

\section{METHODS AND MATERIALS}

The weld image classification system uses two stages; feature extraction and classification which are shown in Figure 1. In feature extraction stage the SURF is applied to the input weld images to extract the features. These extracted features are stored in the database and used for classification. The classification of defect and no defect weld images are made by AEC. 


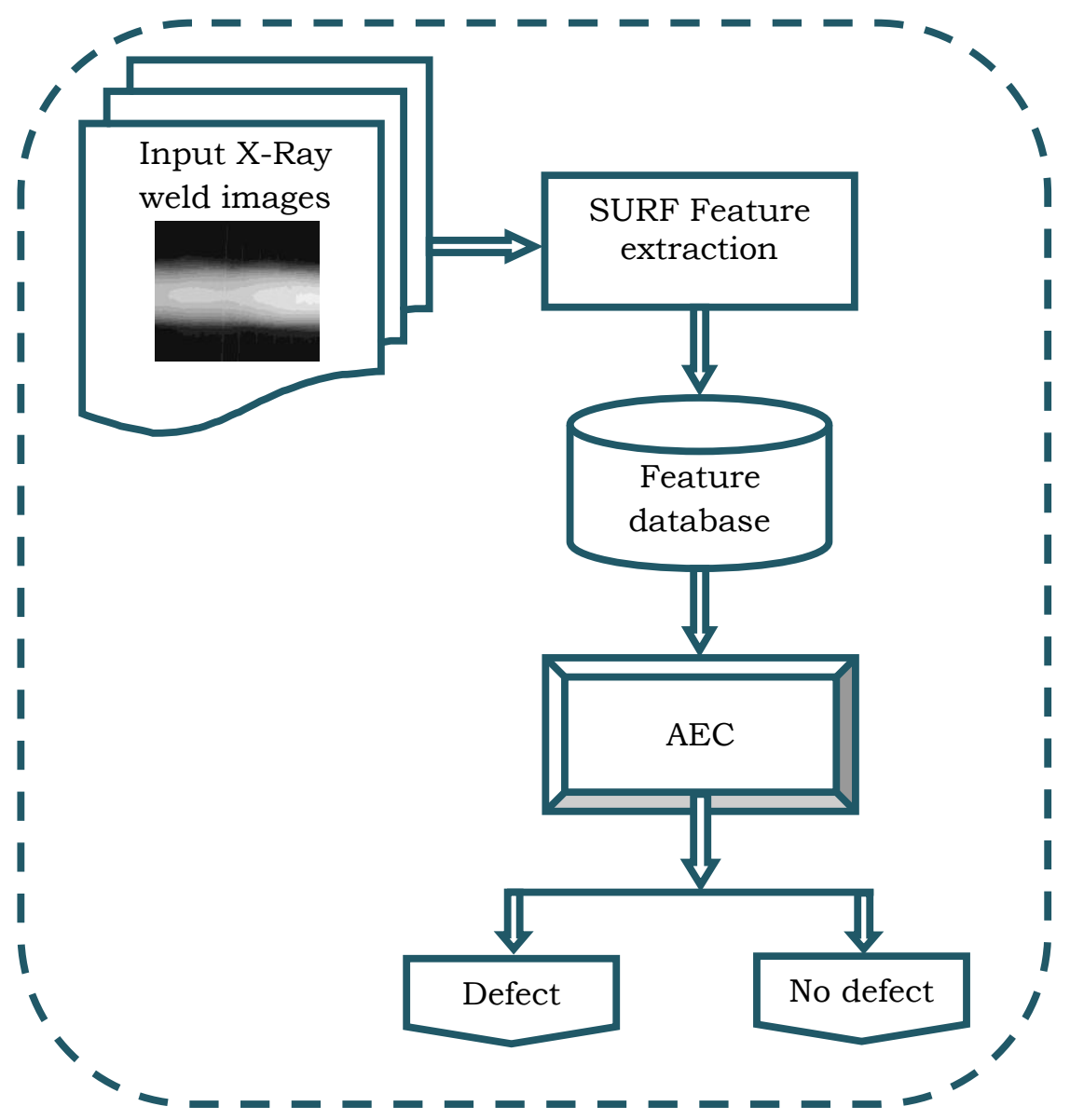

Fig. 1 Weld image classification system using SURF and autoencoder classifier

\section{A. SURF Feature Extraction}

SURF is a local feature descriptor or detector in computer vision. It is stimulated by SIFT. It is the standard version and faster than SIFT. It is also used in the classification of high resolution SAR images [13] and object tracking system [14]. It has better performance in computation time and accuracy. SURF is a determinant of Hessian matrix. Let us consider point, $U(u, v)$ in an image $L$, the Hessian matrix $F(U, \delta)$ in $U$ at scale $\delta$ is defined by,

$$
F(U, \delta)=\left[\begin{array}{l}
K_{u u}(U, \delta) K_{u v}(U, \delta) \\
K_{u v}(U, \delta) K_{v v}(U, \delta)
\end{array}\right]
$$

where $K_{u u}(U, \delta)$ refers to Gaussian second order derivative $\frac{\partial^{2}}{\partial u^{2}} \operatorname{gau}(\delta)$ with the image $L$ at point $U$, where $\operatorname{gau}(\delta)=\frac{1}{2 \pi \delta^{2}} e^{-\left(u^{2}+v^{2}\right) / 2 \delta^{2}}$. The same procedure is used for $K_{u v}(U, \delta)$ and $K_{v v}(U, \delta)$. SURF compares the same type of features and contrast in the matching stage. Figure 2 shows the matching stage in SURF. 


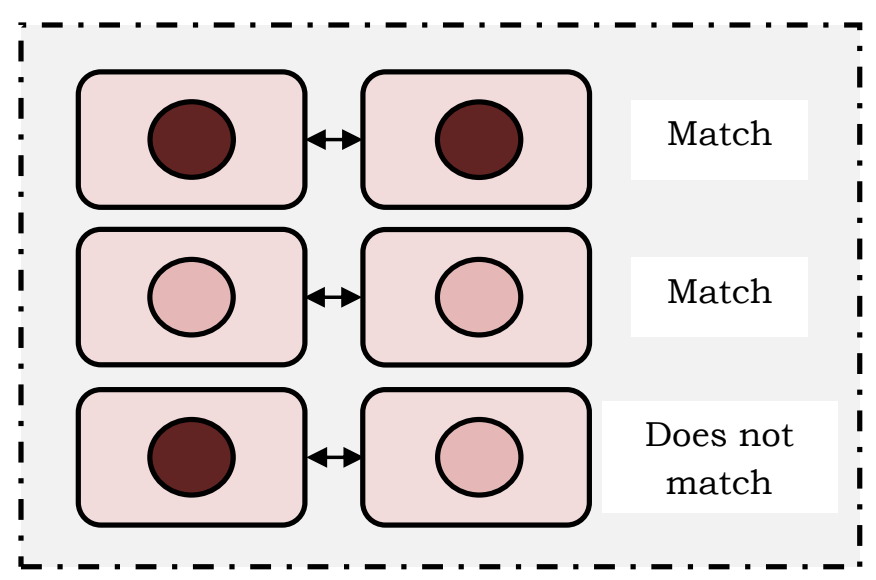

Fig. 2 SURF matching stage

\section{B. Autoencoder Classification}

Autoencoder is a type of ANN to learn about a well-organized data. Autoencoder copy its input to its output. The hidden layer which is presented in the autoencoder has two main parts; encoder and decoder. Encoder maps the given input and decoder maps the encoded data to a reconstruction of original input. Figure 3 shows the encoder and decoder operation in AEC.

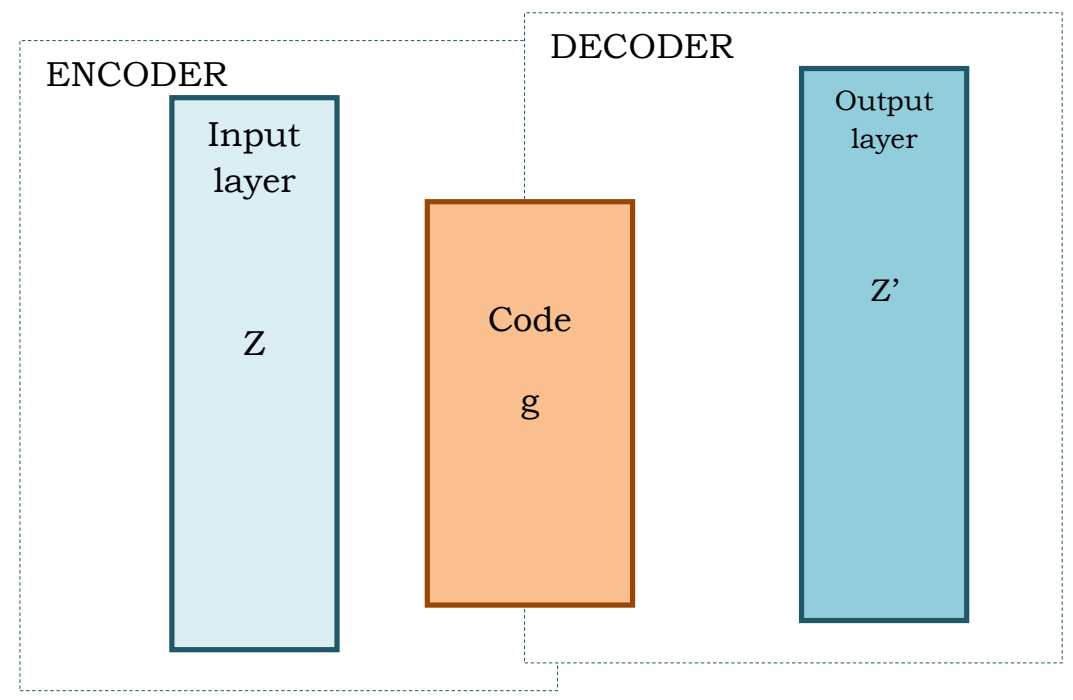

Fig. 3 Encoder and decoder operations in AEC

The two parts of AEC are encoder and decoder. They are defined by $\varphi$ and $\varsigma$, such that:

$$
\begin{gathered}
\varphi: Z \rightarrow P \\
\varsigma: P \rightarrow Z \\
\varphi, \varsigma=\underset{\varphi, \varsigma}{\arg \min }\|X-(\varphi \circ \varsigma) Z\|^{2}
\end{gathered}
$$


In a simple case, the encoder stage of an autoencoder takes the input and maps the function for a single hidden layer. AEC is also used in hyper-spectral image classification [15] and classification of human activity [16]. In this study, the AEC is used for the classification of weld images. It performs classification by three hidden layers with the number of epochs for better classification accuracy.

\section{RESULTS AND DISCUSSION}

The weld image classification system uses GD X-ray weld image database for performance evaluation. From each original image in the GD X-ray database, 256x256 sub-images are extracted for training and testing the AEC classifier as the database contains only 10 images with different defects. From the vast number of sub-images, 120 images (60 normal and 60 defect images) are randomly chosen for the classification. Figure 4 shows the sample normal and defected weld images in the database. Table 1 shows the classification accuracies of the system obtained by AEC with two and three hidden layers.
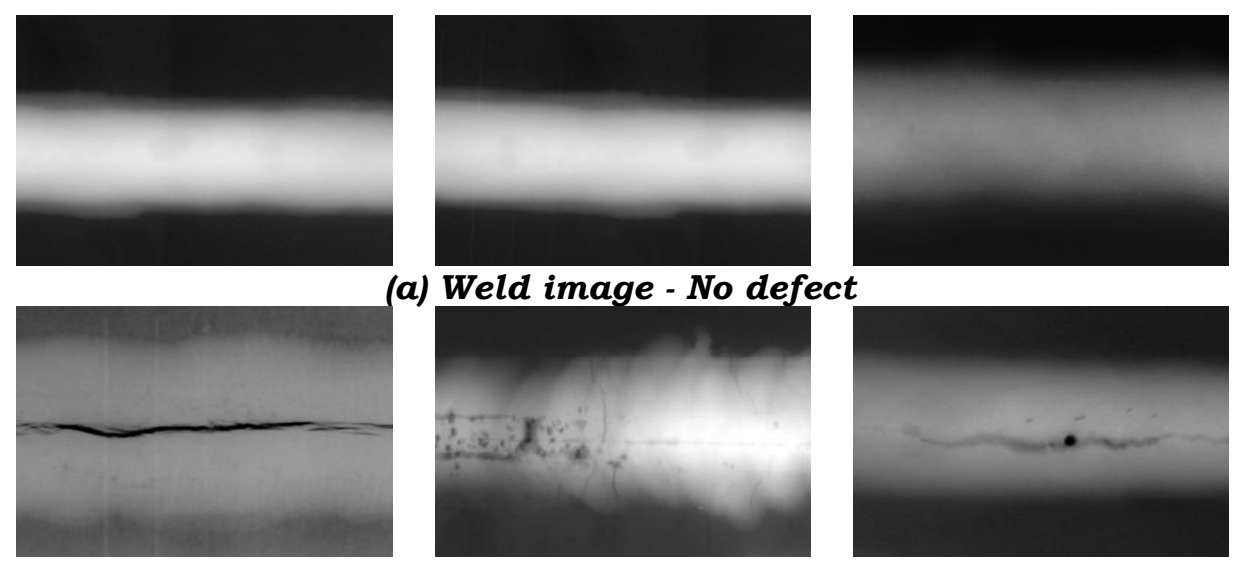

(a) Weld image - No defect

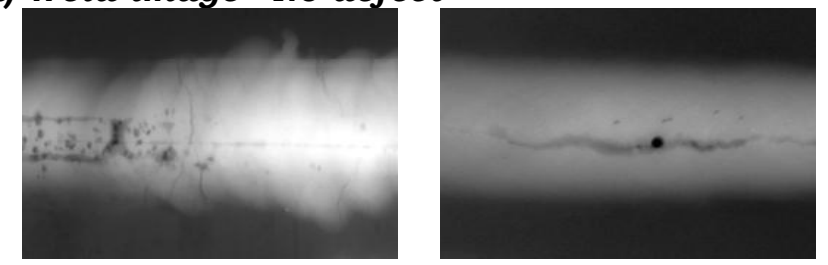

(b) Weld image - Defect

Fig. 4 Sample weld images in the database

TABLE 1 Classification accuracies of weld image classification system using auto-encoder classifier

\begin{tabular}{|c|c|c|c|c|}
\hline \multirow[b]{2}{*}{$\begin{array}{l}\text { Number of } \\
\text { epochs }\end{array}$} & \multicolumn{4}{|c|}{ Classification Accuracy (\%) } \\
\hline & $\begin{array}{c}1^{\text {st }} \text { hidden } \\
\text { layer }\end{array}$ & $\begin{array}{c}2^{\text {nd }} \text { hidden } \\
\text { layer }\end{array}$ & $\begin{array}{c}3^{\text {rd }} \text { hidden } \\
\text { layer }\end{array}$ & $\begin{array}{c}\text { Accuracy } \\
(\%)\end{array}$ \\
\hline 98 & \multirow{2}{*}{100} & 65 & - & 90.50 \\
\hline 97 & & 75 & - & 92.00 \\
\hline 102 & \multirow{3}{*}{150} & 65 & - & 91.50 \\
\hline 100 & & 75 & - & 93.00 \\
\hline 108 & & 85 & - & 92.50 \\
\hline 96 & \multirow{2}{*}{100} & 65 & 70 & 93.00 \\
\hline 99 & & 75 & 80 & 95.00 \\
\hline 110 & \multirow{3}{*}{150} & 65 & 70 & 94.50 \\
\hline 112 & & 75 & 80 & 98.00 \\
\hline 105 & & 85 & 90 & 96.00 \\
\hline
\end{tabular}


From the performance analysis of it is observed that the system correctly classifies the defects by $98 \%$ while using three hidden layers with 150,70 and 80 neurons in the successive hidden layers respectively. The accuracy of the system increases when the number of neurons increases from 100 to 150 . But, this is not true for the second hidden layer. For two hidden layers AEC, the system achieves $93 \%$ accuracy.

\section{CONCLUSION}

An efficient method for weld image classification using machine learning algorithm is presented. The GD X-ray image database is used for weld image classification. Features are extracted by using SURF technique which is a local descriptor. It uses Gaussian smoothing with square shaped filters instead of cascaded filters in SIFT which decreases the computational complexity. The AEC with two and three hidden layers are used for the classification. The performance of the SURF and AEC based system for weld image classification is analyzed by varying the number of neurons in each hidden layers. Experimental results show that $98 \%$ accuracy obtained while using three hidden layers in AEC with different neurons in each hidden layers.

\section{REFERENCES}

[1]. A. Narayanamoorthy, X. Peng, and H. Tang, "Applying low rank representation based spatial pyramid matching in welding image classification", IEEE International Conference on Cybernetics and Intelligent Systems and IEEE Conference on Robotics, Automation and Mechatronics, 2015, pp. 208-211.

[2]. A. Khumaidi, E.M. Yuniarno, and M.H. Purnomo, "Welding defect classification based on convolution neural network (CNN) and Gaussian kernel", International Seminar on Intelligent Technology and Its Applications, 2017, pp. 261-265.

[3]. G. Fei, and W. Kehong, "The research of classification method of arc welding pool image based on invariant moments", IEEE Power Engineering and Automation Conference, Vol. 3, 2011, pp. 73-76.

[4]. A.A. Moghaddam, "Image processing techniques for classification of linear welding defects", International Conference on Knowledge-Based Engineering and Innovation, 2015, pp. 978-981.

[5]. M. Sundaram, J.P. Jose, and G. Jaffino, "Welding defects extraction for radiographic images using $\mathrm{C}$-means segmentation method", International Conference on Communication and Network Technologies, 2014, pp. 79-83.

[6]. F. Mekhalfa, and N. Nacereddine, "Multiclass classification of weld defects in radiographic images based on support vector machines", International Conference on Signal-Image Technology and Internet-Based Systems, 2014, pp. 1-6.

[7]. F. Mekhalfa, and N. Nacereddine, "Gentle Adaboost algorithm for weld defect classification", Signal Processing: Algorithms, Architectures, Arrangements, and Applications, 2017, pp. 301-306.

[8]. J. Hassan, A.M. Awan, and A. Jalil, "Welding defect detection and classification using geometric features", International Conference on Frontiers of Information Technology, 2012, pp. 139-144. 
[9]. J. Kumar, R.S. Anand, and S.P. Srivastava, "Flaws classification using ANN for radiographic weld images", International Conference on Signal Processing and Integrated Networks, 2014, pp. 145-150.

[10]. A. Muniategui, B. Hériz, L. Eciolaza, M. Ayuso, A. Iturrioz, I. Quintana, and P. Álvarez, "Spot welding monitoring system based on fuzzy classification and deep learning", International Conference on Fuzzy Systems, 2017, pp. 1-6.

[11]. D. Gao, Y.X. Liu, and X.G. Zhang, "Binary-tree multi-classifier for welding defects and its application based on SVM", World Congress on Intelligent Control and Automation, Vol. 2, 2006, pp. 8509-8513.

[12]. Y. Hanbing, Z. Lina, and J. Hui, "Research on SVM Based Classification for Welding Defects in Radiographic Testing", International Congress on Image and Signal Processing, 2009.

[13]. X. Tian, C. Wang, and H. Zhang, "Extraction of object features from high resolution SAR images based on SURF features", IEEE International Geoscience and Remote Sensing Symposium, 2011, pp. 1802-1805.

[14]. Y. Sakai, T. Oda, M. Ikeda, and L. Barolli, "An object tracking system based on sift and surf feature extraction methods", International Conference on Network-Based Information Systems, 2015, pp. 561-565.

[15]. Y. Sun, J. Li, W. Wang, A. Plaza, and Z. Chen, "Active learning based autoencoder for hyperspectral imagery classification", International Geoscience and Remote Sensing Symposium, 2016, pp. 469-472.

[16]. H. Badem, A. Caliskan, A. Basturk, and M.E. Yuksel, "Classification of human activity by using a Stacked Autoencoder", Medical Technologies National Congress, 2016, pp. 1-4. 